\title{
Jet Bundles in Quantum Field Theory: The BRST-BV method
}

\author{
Paul McCloud \\ Department of Mathematics, Kings College London
}

May 4, 2018

\begin{abstract}
The geometric interpretation of the Batalin-Vilkovisky antibracket as the Schouten bracket of functional multivectors is examined in detail. The identification is achieved by the process of repeated contraction of even functional multivectors with fermionic functional 1-forms. The classical master equation may then be considered as a generalisation of the Jacobi identity for Poisson brackets, and the cohomology of a nilpotent even functional multivector is identified with the BRST cohomology. As an example, the BRST-BV formulation of gauge fixing in theories with gauge symmetries is reformulated in the jet bundle formalism.
\end{abstract}




\section{Introduction}

The jet bundles to a manifold provide a geometric formulation of the theory of partial differential equations. Coordinates on the jet bundle are given by the independent variables, the dependent variables, and the derivatives of the dependent variables with respect to the independent variables. PDEs are identified with submanifolds of the jet bundle, (infinitesimal) symmetries of the PDEs then occur naturally as tangent vector fields on the jet bundle with flows preserving the submanifolds, [1, chapter 3]. Similarly, symmetries of the action in the Lagrangian formulation of classical field theory occur as evolutionary vector fields on the jet bundle, [2, 1, chapters 4 and 5].

The location of symmetries in the classical field theory reduces to a geometric procedure on the jet bundle. The question remains: can the symmetries be maintained in the quantum field theory? The BRST-BV approach to this question is to consider an extended classical action, dependent on the bosonic fields (only bosonic fields are considered in this article, though the extension to include fermionic fields is clear) and also the external fermionic antifields, B, 何. When the extended action satisfies the classical master equation, the Green's functions for symmetric sources are independent of the antifields up to anomalous terms. One consequence of this is that, in the absence of anomaly terms, the quantisation procedure may be formulated so that the symmetry is preserved in the quantum effective action. Another consequence is the formulation of gauge fixed quantum field theories.

It is natural to consider the jet formulation of the BRST-BV method. The series expansion of a local functional of the fermionic antifield corresponds to a sum of repeatedly contracted functional multivectors on the jet bundle. Moreover, the antifield formalism is valid only on vector bundles, whilst the jet bundle formulation naturally generalises to any bundle, in particular to sigma models.

The first section of this article constitutes a brief introduction to the theory of jet bundles, a more complete review may be found in [1, 2]. The jet bundle analysis of Lagrangian dynamical systems has the advantage of explicitly maintaining locality, reducing a variational problem to a system of PDE's. Functional multivectors are introduced and their basic properties considered. Functional bivectors have been employed in Hamiltonian theory, see [5, 6, 4, 8, 9] and chapters 6 and 7 of [1]. The cohomology of nilpotent bivectors is analysed in the finite dimensional case in 10, 11. The generalisation to arbitrary even functional multivectors considered in this article does not present any extra difficulties. Local bosonic functionals of a fermionic field may then be constructed as repeatedly contracted even functional multivectors.

The next section developes the classical and quantum theories in the jet formalism, in a very general setting, and compares this with the BRST-BV field-antifield formalism, 3, 4, 12, 13, 14, 15, 16]. The jet formulation of the quantum field theory is valid whenever a definition of the functional integral exists. In particular nonlinear bundles, where no global definition of the antifields 
exists, present no difficulties in the jet approach. Instead of considering local functionals of the bosonic field and its fermionic antifield, an even functional multivector is repeatedly contracted with a fermionic functional 1-form. This fermionic functional 1-form generalises both the antifields and the gauge fixing fermion in one object. The classical master equation then becomes a nilpotency condition on the even functional multivector.

The anomalies of the system are interpreted as odd functional multivectors. The Lam action principle 17, 18] may be constructed for the quantum effective action. This is then used to generate the anomaly consistency condition, 19, 20, 21, 221. In the jet formalism, the consistency condition implies that the anomalies define elements of the odd cohomology group of the nilpotent even functional multivector.

The final section analyses the problem of gauge fixing in the jet formalism. The gauge symmetries naturally define a functional bivector that in turn may be used to generate the symmetries, and the algebra the symmetries form defines a nilpotent functional bivector. A combination of these functional bivectors solves the classical master equation and may be used to extend the classical action. It is observed that the fermionic functional 1-form contains both the gauge fixing fermion and the ghost field in one object, and in this way the jet formalism reproduces the BRST-BV results, [3, 1, 19, 21].

A future paper will consider the use of jet bundles in the analysis of chiral and $\mathrm{W}$-symmetry algebras and the anomalies in these algebras. This requires an extension of the jet bundle theory to bundles with a chiral decomposition of the tangent bundle. W-symmetries are discovered, under special conditions, in arbitrary dimensions, and the nature of the full Lie algebra generated from the $\mathrm{W}$-algebra is revealed. The classical master equation is solved, thus defining a cohomology problem for the calculation of anomalies in chiral and W-symmetry algebras.

\section{Introduction to jet bundles and functional mul- tivectors}

\section{$2.1 \quad$ Jet bundles}

The notion of the tangent vector to a manifold arises by considering the first order contact of curves in the manifold. The theory of jets generalises this in two ways: the contact of higher dimensional curves, i.e. submanifolds, and contact at arbitrarily high orders are considered. This leads to the definition of the jet bundles of a manifold. The jet bundles form the natural geometric arena for the study of partial differential equations, and in particular the equations arising from the Lagrangian approach to field theory.

The jet bundles to a manifold, even a trivial manifold, have non-trivial geometry. For a fibre bundle, a restricted jet bundle may be defined by restricting 
attention to submanifolds occuring as the images of sections of the bundle. This simplification neglects the possibility of singular and multi-valued solutions of PDEs, accommodated in the full jet bundle. However, it is sufficient for the purposes of this article.

Consider a fibre bundle $B \stackrel{\pi}{\rightarrow} M$ over the compact, connected, and orientable manifold without boundary $M$. Denote the space of smooth sections $\phi: M \rightarrow B$ of $B \stackrel{\pi}{\rightarrow} M$ by $\Gamma(B \stackrel{\pi}{\rightarrow} M)$ or $\Gamma(\pi)$, and the space of local sections near $x_{0} \in M$ by $\Gamma_{x_{0}}(\pi)$. In terms of the local adapted coordinates $\left(x^{i}, \phi^{\alpha}\right)$ on $B$, the two local sections $\phi_{1}, \phi_{2} \in \Gamma_{x_{0}}(\pi)$ are equivalent under the relation of infinite order contact at $x_{0}$ if:

$$
\left.\frac{\partial^{|I|}\left(\phi^{\alpha} \circ \phi_{1}\right)}{\partial x^{I}}\right|_{x_{0}}=\left.\frac{\partial^{|I|}\left(\phi^{\alpha} \circ \phi_{2}\right)}{\partial x^{I}}\right|_{x_{0}}
$$

for all multi-indices $I=\left(n_{1}, \ldots, n_{m}\right)$, where $m=\operatorname{dim} M$. The jet space $J_{x_{0}}^{\infty} \pi$ is the space $\Gamma_{x_{0}}(\pi)$ modulo this relation, and the collection $J^{\infty} \pi=\bigcup_{x \in M} J_{x}^{\infty} \pi$ constitutes the jet bundle over $M, J^{\infty} \pi \stackrel{\pi_{\infty}}{\rightarrow} M$. Local coordinates on the jet bundle are given by:

$$
\phi_{I}^{\alpha}\left([\phi]_{x_{0}}\right)=\left.\frac{\partial^{|I|}\left(\phi^{\alpha} \circ \phi\right)}{\partial x^{I}}\right|_{x_{0}}
$$

Any section $\phi \in \Gamma(\pi)$ may be prolonged to a section $j^{\infty} \phi$ of the jet bundle via $j^{\infty} \phi(x)=[\phi]_{x}$. A function $f$ on $J^{\infty} \pi$ can then be evaluated on sections of $B \stackrel{\pi}{\rightarrow} M$ as a function on the base space. The total derivatives $d_{i}$, tangent vectors on $J^{\infty} \pi$, are defined by the expression:

$$
\left(d_{i} f\right) \circ j^{\infty} \phi=\partial_{i}\left(f \circ j^{\infty} \phi\right)
$$

In terms of local coordinates:

$$
d_{i}=\partial_{i}+\phi_{I i}^{\alpha} \frac{\partial}{\partial \phi_{I}^{\alpha}}
$$

The total derivatives determine a horizontal subspace to the tangent bundle of $J^{\infty} \pi$. Since the total derivatives commute, $\left[d_{i}, d_{j}\right]=0$, this defines a canonical integrable connection on the jet bundle:

$$
V J^{\infty} \pi=<\frac{\partial}{\partial \phi_{I}^{\alpha}}>, H J^{\infty} \pi=<d_{i}>
$$

\subsection{Variational bicomplex}

The decomposition of the tangent bundle induces a decomposition of the spaces of differential forms on the jet space. Denote the space of forms with $r$ horizontal and $s$ vertical factors by $\Omega_{s}^{r} \pi$. The exterior derivative decomposes, $d=d_{h}+d_{v}$, where:

$$
d_{h}=d x^{i} d_{i}, d_{v}=\left(d \phi_{I}^{\alpha}-\phi_{I i}^{\alpha} d x^{i}\right) \frac{\partial}{\partial \phi_{I}^{\alpha}}
$$




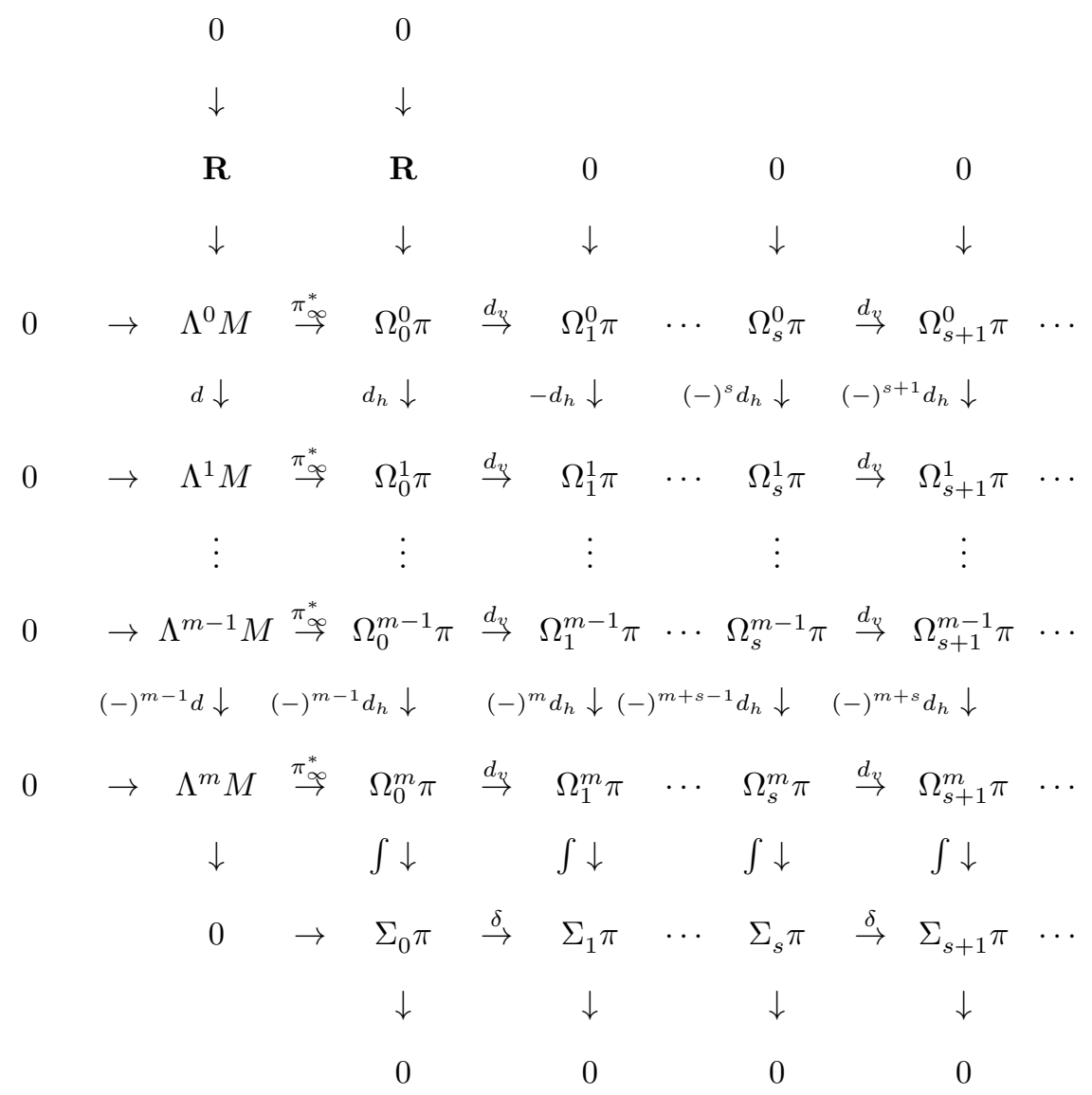

Figure 1: The variational bicomplex

The properties of the horizontal and vertical exterior derivatives are summarised in the variational bicomplex, Figure 11. This diagram commutes, and any row or column forms a complex. Furthermore, it can be demonstrated that the variational bicomplex is locally exact - on topologically trivial spaces the cohomology of the variational bicomplex is trivial.

The bicomplex is closed on the first column by the de Rham complex of the base space, and on the bottom row by the variational complex:

$$
0 \quad \rightarrow \quad \Sigma_{0} \pi \quad \stackrel{\delta}{\rightarrow} \quad \Sigma_{1} \pi \quad \cdots \quad \Sigma_{s} \pi \quad \stackrel{\delta}{\rightarrow} \Sigma_{s+1} \pi \quad \cdots
$$

The functional $s$-forms $\Sigma_{s} \pi$ are given by the quotient:

$$
\Sigma_{s} \pi=\Omega_{s}^{m} \pi / d_{h} \Omega_{s}^{m-1} \pi
$$


The projection $\Omega_{s}^{m} \pi \rightarrow \Sigma_{s} \pi$ is denoted by the integral sign $\int$, and the variational derivative $\delta$ is induced by the vertical exterior derivative:

$$
\delta \int \omega=\int d_{v} \omega
$$

Define the variational cohomology groups:

$$
H_{s}(\pi, \delta)=\operatorname{Ker} \delta \cap \Sigma_{s} \pi / \operatorname{Im} \delta \cap \Sigma_{s} \pi
$$

The vertical vector fields may be contracted with forms on the jet space, reducing the vertical degree by one. It is of interest to consider when it is possible to contract vertical vector fields with functional forms in an unambiguous manner. Take $\left.\eta=\eta^{i} \wedge d_{i}\right\lrcorner \Omega \in \Omega_{s}^{m-1} \pi$, with $\eta^{i} \in \Omega_{s}^{0} \pi$, and $v$ a vertical vector field. Then:

$$
\left.\left.v\lrcorner d_{h} \eta+d_{h}(v\lrcorner \eta\right)=(-)^{s}\left[v, d_{i}\right]\right\lrcorner \eta^{i} \wedge \Omega
$$

In particular, when $\left[v, d_{i}\right]=0$ then $\left.\left.v\right\lrcorner d_{h} \eta=-d_{h}(v\lrcorner \eta\right)$. Vertical vector fields satisfying $\left[v, d_{i}\right]=0$ are evolutionary vector fields, and take the form:

$$
v=\left(d_{I} v^{\alpha}\right) \frac{\partial}{\partial \phi_{I}^{\alpha}}
$$

for some $v^{\alpha}$. Thus evolutionary vector fields may be contracted with functional forms, $\left.v\lrcorner \int \omega=\int v\right\lrcorner \omega$.

The representative for a functional form is only defined up to a total horizontal derivative. Integration by parts may thus be performed under the integral sign. In particular, this may be employed to reduce functional forms to canonical forms. Take a volume form $\Omega=\rho d^{m} x$ on $M$. Using integration by parts, functional 1-forms may be expressed in the canonical form $\int \omega_{\alpha} d \phi^{\alpha} \wedge \Omega$. In particular:

$$
\delta \int \mathcal{L} \Omega=\int E_{\alpha}(\mathcal{L}) d \phi^{\alpha} \wedge \Omega
$$

where $E_{\alpha}$ is the Euler operator:

$$
E_{\alpha}=(-)^{|I|} \rho^{-1} d_{I} \rho \frac{\partial}{\partial \phi_{I}^{\alpha}}
$$

Thus $\delta \int \mathcal{L} \Omega=0$ if and only if $E_{\alpha}(\mathcal{L})=0$.

\subsection{Evaluation and expansion of functionals}

Many of the properties of the variational cohomology have parallels in the finite dimensional de Rham cohomology, with functional forms replaced by differential forms, and evolutionary vector fields replaced with tangent vector fields. In fact (at least locally) functional forms may be identified with forms on the space 
of sections $\Gamma(\pi)$, and evolutionary vector fields with tangent vector fields on $\Gamma(\pi)$. The Taylor series expansion of functional 0-forms may thus be considered analogously to the expansion of functions on manifolds.

Horizontal forms on the jet bundle may be evaluated on sections as forms on the base space. By definition of the total derivatives, $d_{h} \eta \circ j^{\infty} \phi=d(\eta \circ$ $j^{\infty} \phi$ ) for $\eta \in \Omega_{0}^{m-1} \pi$. Since $M$ is compact and boundary-less, then by Stokes' theorem functional 0 -forms may be evaluated unambiguously on sections-for $S=\int \mathcal{L} \Omega \in \Sigma_{0} \pi$ then:

$$
S[\phi]=\int_{M} \mathcal{L} \circ j^{\infty} \phi \Omega
$$

The functional 0-forms constitute the local functionals in $\Lambda^{0} \Gamma(\pi)$.

The tangent vectors on $\Gamma(\pi)$ are transformations $\delta \phi^{\alpha}=v^{\alpha}$, and are identified with evolutionary vector fields. The requirement that evolutionary vector fields commute with the total derivatives thus corresponds to the rule $\left[\delta, d_{i}\right]=0$ for transformations. In particular, for $\int \omega \in \Sigma_{s} \pi$ and $v_{1}, \ldots, v_{s}$ evolutionary vector fields, then $\left.\left(v_{1}, \ldots, v_{s}\right)\right\lrcorner \int \omega$ is a local functional. Thus the functional $s$-forms constitute the local $s$-forms on $\Gamma(\pi)$.

The transformation $\delta \phi^{\alpha}=v^{\alpha}$ may be exponentiated to give a flow $e^{\lambda v}$ on $\Gamma(\pi)$. The variational derivative of a functional $S$ is defined by:

$$
\frac{d}{d \lambda} S\left[e^{\lambda v} \phi_{0}\right]=\int v^{\alpha} \frac{\delta S}{\delta \phi^{\alpha}} \Omega\left[e^{\lambda v} \phi_{0}\right]
$$

For the local functional $S=\int \mathcal{L} \Omega$, then $\delta S / \delta \phi^{\alpha}=E_{\alpha}(\mathcal{L})$. The Taylor series expansion of $S$ is thus:

$$
\begin{aligned}
S\left[e^{\lambda v} \phi_{0}\right] & \left.=\sum_{n=0}^{\infty} \frac{\lambda^{n}}{n !}(v\lrcorner \delta\right)^{n} S\left[\phi_{0}\right] \\
& \left.=S\left[\phi_{0}\right]+\sum_{n=0}^{\infty} \frac{\lambda^{n+1}}{(n+1) !} v\right\lrcorner v^{n}(\delta S)\left[\phi_{0}\right]
\end{aligned}
$$

When $B \stackrel{\pi}{\rightarrow} M$ is a vector bundle, take the transformation $v$ to be a translation. Then the expansion above for a local functional gives:

$$
S[\phi]=S[0]+\sum_{n=0}^{\infty} \frac{1}{(n+1) !} \int \phi^{\alpha} \phi_{I_{1}}^{\beta_{1}} \ldots \phi_{I_{n}}^{\beta_{n}} S_{\alpha_{\beta_{1} \ldots \beta_{n}}}^{I_{1} \ldots I_{n}} \Omega
$$

with coefficient functions:

$$
S_{\alpha_{\beta_{1} \ldots \beta_{n}}}^{I_{1} \ldots I_{n}}=\left.\frac{\partial^{n}}{\partial \phi_{I_{1}}^{\beta_{1}} \ldots \partial \phi_{I_{n}}^{\beta_{n}}}\left(\frac{\delta S}{\delta \phi^{\alpha}}\right)\right|_{\phi=0}
$$

The expansion of the classical action defines the propagators and vertices of the perturbative quantum field theory, in which case the coefficient functions above 
would be renormalised in order to maintain finiteness. The above expansion may also be employed for chiral local functionals to give a basis for the full Lie algebra generated from a $\mathrm{W}$-algebra. This will be explained in a future paper.

\subsection{Functional multivectors}

The parallel between variational cohomology and de Rham cohomology fails when considering the wedge product of differential forms. The wedge product of two local functional forms is bilocal, and hence the space of functional forms does not close under the product - the functional forms do not possess a wedge product structure. The definition of a dual space to the functional forms is thus ambiguous. The functional $s$-forms $\Sigma_{s} \pi$ define alternating, $s$-linear maps on the evolutionary vector fields, valued in the space of local functionals $\Sigma_{0} \pi$. Define the dual space $\Sigma^{s} \pi$ of functional $s$-vectors to be the space of alternating, $s$-linear maps $\left(\Sigma_{1} \pi\right)^{s} \rightarrow \Sigma_{0} \pi$. For completion, define also the functional 0-vectors $\Sigma^{0} \pi$ to be the local functionals $\Sigma_{0} \pi$.

Since the canonical form for $\omega \in \Sigma_{1} \pi$ is $\omega=\int \omega_{\alpha} d \phi^{\alpha} \wedge \Omega$, the functional $s$-vector $v \in \Sigma^{s} \pi$ may be written:

$$
v=\int \Omega_{1} \ldots \int \Omega_{s} v^{\alpha_{1} \ldots \alpha_{s}} \frac{\partial}{\partial \phi_{1}^{\alpha_{1}}} \wedge \ldots \wedge \frac{\partial}{\partial \phi_{s}^{\alpha_{s}}}
$$

with the obvious definition of contraction with functional 1-forms. The result of contraction must be a local functional. The coefficient functions must thus be products of $\delta$-functions, of the form:

$$
v^{\alpha_{1} \ldots \alpha_{s}}=\int(-)^{\left|I_{1}\right|+\cdots+\left|I_{s}\right|} v^{\alpha_{1} I_{1} \ldots \alpha_{s} I_{s}} \rho_{1}^{-1} d_{I_{1}}^{(1)}\left(\rho_{1} \delta_{x}\left(x_{1}\right)\right) \ldots \rho_{s}^{-1} d_{I_{s}}^{(s)}\left(\rho_{s} \delta_{x}\left(x_{s}\right)\right) \Omega
$$

where the $\delta$-function satisfies $f(x)=\int f(y) \delta_{x}(y) \Omega_{y}$. Performing the integrals gives the general form of the functional $s$-vector:

$$
v=\int \Omega v^{\alpha_{1} I_{1} \ldots \alpha_{s} I_{s}} d_{I_{1}} \frac{\partial}{\partial \phi^{\alpha_{1}}} \wedge \ldots \wedge d_{I_{s}} \frac{\partial}{\partial \phi^{\alpha_{s}}}
$$

so that for the functional 1-form $\omega=\int \omega_{\alpha} d \phi^{\alpha} \wedge \Omega$ :

$$
v\lrcorner \omega=\sum_{r=1}^{s}(-)^{r-1} \int \Omega v^{\alpha_{1} I_{1} \ldots \alpha_{s} I_{s}}\left(d_{I_{r}} \omega^{\alpha_{r}}\right) d_{I_{1}} \frac{\partial}{\partial \phi^{\alpha_{1}}} \wedge \ldots \wedge d_{I_{s}} \frac{\partial}{\partial \phi^{\alpha_{s}}}
$$

where the wedge product in the above expression omits the $r$ th factor.

The coordinate representation above for the functional $s$-vector $v$ is not unique. As for functional forms, integration by parts may be performed under the integral sign. Functional multivectors may thus be reduced to canonical forms - the functional vectors have the canonical form:

$$
\int \Omega v^{\alpha} \frac{\partial}{\partial \phi^{\alpha}}
$$


and are identified with the evolutionary vector fields, whilst the functional bivectors have the canonical form:

$$
\frac{1}{2} \int \Omega \frac{\partial}{\partial \phi^{\alpha}} \wedge D^{\alpha \beta} \frac{\partial}{\partial \phi^{\beta}}
$$

for some skew adjoint operator $D^{\alpha \beta}$.

Using the coordinate expression for the functional $s$-vector $v$, the variational derivative $\delta v$ may be defined:

$$
\delta v=\int d \phi_{J}^{\beta} \wedge \Omega \frac{\partial v^{\alpha_{1} I_{1} \ldots \alpha_{s} I_{s}}}{\partial \phi_{J}^{\beta}} d_{I_{1}} \frac{\partial}{\partial \phi^{\alpha_{1}}} \wedge \ldots \wedge d_{I_{s}} \frac{\partial}{\partial \phi^{\alpha_{s}}}
$$

This variational derivative does not have a coordinate independent meaning (without the introduction of a connection on the space of sections $\Gamma(\pi)$ ), and cannot be extended to a global definition. However, the combination:

$$
\left.\left.\left[v_{1}, v_{2}\right]=v_{1}\right\lrcorner \delta v_{2}+(-)^{s_{1} s_{2}} v_{2}\right\lrcorner \delta v_{1} \in \Sigma^{s_{1}+s_{2}-1} \pi
$$

for the functional multivectors $v_{1,2} \in \Sigma^{s_{1}, s_{2}} \pi$ is globally defined, and defines the Schouten bracket of $v_{1}$ and $v_{2}$. The bracket is 'antisymmetric':

$$
\left[v_{1}, v_{2}\right]=(-)^{s_{1} s_{2}}\left[v_{2}, v_{1}\right]
$$

and satisfies the 'Jacobi identity':

$$
(-)^{s_{1}\left(s_{3}+1\right)}\left[v_{1},\left[v_{2}, v_{3}\right]\right]+\text { cyclic permutations }=0
$$

for $v_{1,2,3} \in \Sigma^{s_{1}, s_{2}, s_{3}} \pi$. Furthermore, if $\omega \in \Sigma_{1} \pi$ is a closed functional 1-form, $\delta \omega=0$, then the bracket satisfies the closed form identity:

$$
\left.\left.\left.\left[v_{1}, v_{2}\right]\right\lrcorner \omega+\left[v_{1}\right\lrcorner \omega, v_{2}\right]+(-)^{s_{1}}\left[v_{1}, v_{2}\right\lrcorner \omega\right]=0
$$

\subsection{Multivector cohomology}

The Schouten bracket of the evolutionary vector field $v$ with the local functional $S$ is simply the variation of $S, v S \equiv v\lrcorner \delta S$, whilst the Schouten bracket of two evolutionary vector fields is the familiar vector field commutator. Consider the interpretation of the bracket for general functional multivectors.

For the even functional multivector $\Gamma \in \bigoplus_{r=0}^{\infty} \Sigma^{2 r} \pi$, the Jacobi identity for the Schouten bracket gives:

$$
[\Gamma,[\Gamma, v]]=-\frac{1}{2}[[\Gamma, \Gamma], v]
$$

for any functional multivector $v$. When $\Gamma$ is nilpotent, $[\Gamma, \Gamma]=0$, the commutator action $\Gamma: v \mapsto[\Gamma, v]$ is nilpotent. In this case, $\Gamma$ defines a complex:

$$
\bigoplus_{r=0}^{\infty} \Sigma^{2 r} \pi \underset{\Gamma}{\stackrel{\Gamma}{\rightleftharpoons}} \bigoplus_{r=0}^{\infty} \Sigma^{2 r+1} \pi
$$


Define the even and odd $\Gamma$-cohomology groups:

$$
\begin{aligned}
H^{\text {even }}(\pi, \Gamma) & =\operatorname{Ker} \Gamma \cap \bigoplus_{r=0}^{\infty} \Sigma^{2 r} \pi / \operatorname{Im} \Gamma \cap \bigoplus_{r=0}^{\infty} \Sigma^{2 r} \pi \\
H^{\text {odd }}(\pi, \Gamma) & =\operatorname{Ker} \Gamma \cap \bigoplus_{r=0}^{\infty} \Sigma^{2 r+1} \pi / \operatorname{Im} \Gamma \cap \bigoplus_{r=0}^{\infty} \Sigma^{2 r+1} \pi
\end{aligned}
$$

Thus nilpotent even functional multivectors define cohomology operators on the functional multivectors, via the Schouten bracket action.

Functional bivectors can be used to construct Poisson brackets on the space of local functionals. Take $\Gamma$ to be a functional bivector, with skew adjoint operator $D^{\alpha \beta}$. The Poisson bracket on $\Sigma_{0} \pi$ determined by $\Gamma$ is:

$$
\{S, T\}=\Gamma\lrcorner \delta S\lrcorner \delta T=\int \frac{\delta S}{\delta \phi^{\alpha}} D^{\alpha \beta} \frac{\delta T}{\delta \phi^{\beta}} \Omega
$$

The nilpotency condition $[\Gamma, \Gamma]=0$ and the Jacobi identity for the Poisson bracket are equivalent:

$$
\left.\left.\left.\{R,\{S, T\}\}+\text { cyclic permutations }=-\frac{1}{2}[\Gamma, \Gamma]\right\lrcorner \delta R\right\lrcorner \delta S\right\lrcorner \delta T
$$

i.e. the Poisson bracket satisfies the Jacobi identity if and only if $\Gamma$ is nilpotent. In this case, the commutator action of $\Gamma$ gives a complex:

$$
0 \quad \rightarrow \quad \Sigma^{0} \pi \quad \stackrel{\Gamma}{\rightarrow} \quad \Sigma^{1} \pi \quad \cdots \quad \Sigma^{s} \pi \quad \stackrel{\Gamma}{\rightarrow} \quad \Sigma^{s+1} \pi \quad \cdots
$$

with cohomology groups:

$$
H^{s}(\pi, \Gamma)=\operatorname{Ker} \Gamma \cap \Sigma^{s} \pi / \operatorname{Im} \Gamma \cap \Sigma^{s} \pi
$$

The cohomology groups may be used to analyse the Poisson bracket, for example the group $H^{0}(\pi, \Gamma)$ is identified with the space of distinguished local functionals, whose Poisson bracket with any other local functional vanishes.

\subsection{Contraction with fermionic functional 1-forms}

The functional multivector, when contracted, is local and antisymmetric in its arguments. This suggests that they may be used to provide an expansion for local functionals of fermionic fields. Effectively, the functional multivectors are identified with the local functionals on a superspace. In fact this is only possible in the case of a linear bundle, but the idea of repeated contraction with fermionic functional 1-forms is valid in nonlinear bundles, and provides a natural generalisation of the notion of the fermionic antifield. 
Let $\eta=\int \eta_{\alpha} d \phi^{\alpha} \wedge \Omega$ be a fermionic functional 1-form. Then $\eta$ may be repeatedly contracted with a functional multivector. For $v=\sum_{s=0}^{\infty} v^{s}, v^{s} \in$ $\Sigma^{s} \pi$, define:

$$
\left.\left.\left.v_{\eta}^{r}=\sum_{s=r}^{\infty} \frac{1}{(s-r) !} v^{s}\right\lrcorner \eta\right\lrcorner \stackrel{s-r}{\cdots}\right\lrcorner \eta \in \Sigma^{r} \pi
$$

The cases $r=0$ and $r=1$ are of particular importance. For $r=0, v_{\eta} \equiv v_{\eta}^{0}$ is a local functional, bosonic if $v$ is even, fermionic if $v$ is odd. For $r=1, \hat{v}_{\eta} \equiv v_{\eta}^{1}$ is an evolutionary vector field.

If $\chi$ is another fermionic functional 1-form, the repeated contractions with the forms $\eta, \chi$, and $\eta+\chi$ may all be defined. Separating out the contractions demonstrates that they are related:

$$
v_{\eta+\chi}^{r}=\sum_{s=r}^{\infty}\left(v_{\eta}^{s}\right)_{\chi}^{r}
$$

If $\chi$ is a perturbation of $\eta$, then to lowest order in $\chi$ :

$$
\left.v_{\eta+\chi}^{r}-v_{\eta}^{r}=v_{\eta}^{r+1}\right\lrcorner \chi
$$

In the case $r=0$ :

$$
\left.v_{\eta+\chi}-v_{\eta}=\hat{v}_{\eta}\right\lrcorner \chi
$$

In the linear case this expression would define the variational derivative of $v_{\eta}$ with respect to the fermionic antifield $\eta$. Thus in the general nonlinear case this is replaced with the evolutionary vector field $\hat{v}_{\eta}$.

When the fermionic functional 1-form $\eta$ is closed, $\delta \eta=0$, the closed form identity can be used to take the contractions into the commutator, resulting in an expression of the form:

$$
\left[v_{1}, v_{2}\right]_{\eta}^{t}=\sum_{r=\max \left\{0, t+1-s_{2}\right\}}^{\min \left\{s_{1}, t+1\right\}} \lambda_{t r}^{s_{1} s_{2}}\left[v_{1 \eta}^{r}, v_{2 \eta}^{t+1-r}\right]
$$

for some coefficients $\lambda_{t r}^{s_{1} s_{2}}$, where $v_{1,2} \in \Sigma^{s_{1}, s_{2}} \pi$. The closed form identity gives the relation:

$$
\left(s_{1}+s_{2}-t\right) \lambda_{(t-1) r}^{s_{1} s_{2}}=(-)^{s_{2}+r+t+1}\left(s_{1}-r\right) \lambda_{t(r+1)}^{s_{1} s_{2}}+(-)^{r+1}\left(s_{2}+r-t\right) \lambda_{t r}^{s_{1} s_{2}}
$$

which is solved to give:

$$
\left[v_{1}, v_{2}\right]_{\eta}^{t}=\sum_{r=\max \left\{0, t+1-s_{2}\right\}}^{\min \left\{s_{1}, t+1\right\}}(-)^{\left(s_{2}+t\right)(r+1)+s_{1}+1}\left[v_{1 \eta}^{r}, v_{2 \eta}^{t+1-r}\right]
$$

The expansion is valid for $0 \leq t \leq s_{1}+s_{2}-1$. In particular, in the $t=0$ case:

$$
\left[v_{1}, v_{2}\right]_{\eta}=(-)^{s_{1}+1}\left\{\hat{v}_{1 \eta}\left(v_{2 \eta}\right)+(-)^{s_{2}} \hat{v}_{2 \eta}\left(v_{1 \eta}\right)\right\}
$$


For the even functional multivector $\Gamma, \Gamma_{\eta}$ is a bosonic local functional, and the expression above gives:

$$
\hat{\Gamma}_{\eta}\left(\Gamma_{\eta}\right)=-\frac{1}{2}[\Gamma, \Gamma]_{\eta}
$$

Thus $\Gamma_{\eta}$ is symmetric under $\hat{\Gamma}_{\eta}$ if and only if $\Gamma$ is nilpotent at $\eta,[\Gamma, \Gamma]_{\eta}=0$.

When the bundle $B \stackrel{\pi}{\rightarrow} M$ is a vector bundle, the fermionic functional 1-form $\eta$ may be taken to be field independent, $\eta=\int \eta_{\alpha}(x) d \phi^{\alpha} \wedge \Omega$ for a fermionic antifield $\eta \in \Gamma\left(B^{*} \rightarrow M\right)$. The functional multivector $v$ is identified with the local functional $v_{\eta}=v[\phi, \eta]$ of the field $\phi$ and its antifield $\eta$, and the Schouten bracket induces the Batalin-Vilkovisky antibracket, $\left[v_{1}, v_{2}\right]_{\eta}=\left(v_{1 \eta}, v_{2 \eta}\right)$, where:

$$
\left(v_{1}, v_{2}\right)=\int\left\{\frac{\delta v_{1}}{\delta \eta_{\alpha}} \frac{\delta v_{2}}{\delta \phi^{\alpha}}+(-)^{s_{1} s_{2}} \frac{\delta v_{2}}{\delta \eta_{\alpha}} \frac{\delta v_{1}}{\delta \phi^{\alpha}}\right\} \Omega
$$

Note the symmetry in this bracket between $\phi$ and $\eta$, in particular the role of the field and the antifield may be interchanged in the vector bundle case. The commutator action of the functional multivector $v$ may be written as an evolutionary vector field on $B \times B^{*} \rightarrow M$ :

$$
s_{v}=\int \Omega\left\{\frac{\delta v}{\delta \eta_{\alpha}} \frac{\partial}{\partial \phi^{\alpha}}+(-)^{s} \frac{\delta v}{\delta \phi^{\alpha}} \frac{\partial}{\partial \eta_{\alpha}}\right\}
$$

For the even functional multivector $\Gamma$, the BRST operator:

$$
s_{\Gamma}=\int \Omega\left\{\frac{\delta \Gamma}{\delta \eta_{\alpha}} \frac{\partial}{\partial \phi^{\alpha}}+\frac{\delta \Gamma}{\delta \phi^{\alpha}} \frac{\partial}{\partial \eta_{\alpha}}\right\}
$$

satisfies:

$$
s_{\Gamma}^{2}=-\frac{1}{2} s_{[\Gamma, \Gamma]}
$$

and so $s_{\Gamma}$ is nilpotent if and only if $\Gamma$ is nilpotent, in which case the $\Gamma$ cohomology on functional multivectors is identified with the $s_{\Gamma}$-cohomology on local functionals of $\phi$ and $\eta$.

\section{The BRST-BV method in the jet bundle for- malism}

\subsection{Symmetry in classical field theory}

The classical dynamics of a field $\phi \in \Gamma(\pi)$ is given, in the Lagrangian formalism, by extremising the classical action $S=\int \mathcal{L} \Omega \in \Sigma_{0} \pi$ over $\Gamma(\pi)$. Thus the classical field equation is:

$$
\delta S[\phi]=0, \text { or } E_{\alpha}(\mathcal{L}) \circ j^{\infty} \phi=0
$$


defining the extremal point of the action. Variations of the field that leave the action invariant define infinitesimal symmetries of $S$. Thus the evolutionary vector field $v$ is a symmetry of the classical action if $v S=0$.

The classical action may have a dependence on external, non-dynamical fields. One example is the class of actions constructed by repeated contraction of the even functional multivector $\Gamma=\sum_{r=0}^{\infty} \Gamma^{2 r}, \Gamma^{2 r} \in \Sigma^{2 r} \pi$. The action is then parametrized by the space of fermionic functional 1-forms. The even functional multivector $\Gamma$ defines not only the action $\Gamma_{\eta}$, for the fermionic functional 1-form $\eta$, but also the evolutionary vector field $\hat{\Gamma}_{\eta}$. A local functional $J \in \Sigma_{0} \pi$ is said to be symmetric under $\Gamma$ at $\eta$ if $[\Gamma, J]_{\eta}=0$, in which case $\hat{\Gamma}_{\eta}(J)=0$. For any closed fermionic functional 1-form $\eta \in \operatorname{Ker} \delta \cap \Sigma_{1} \pi$ :

$$
\hat{\Gamma}_{\eta}\left(\Gamma_{\eta}\right)=-\frac{1}{2}[\Gamma, \Gamma]_{\eta}
$$

so that $\hat{\Gamma}_{\eta}$ is a symmetry of the action $\Gamma_{\eta}$ when $\Gamma$ satisfies the classical master equation at $\eta$ :

$$
[\Gamma, \Gamma]_{\eta}=0
$$

i.e. $\Gamma$ is nilpotent at $\eta$.

In practice, actions defined by even functional multivectors arise by considering the extension of the original action $S$ to include terms defining an algebra of symmetries of $S$. Suppose the action is symmetric under the even functional multivector $v=\sum_{r=1}^{\infty} v^{2 r}, v^{2 r} \in \Sigma^{2 r} \pi$, i.e. $[v, S]=0$. The extension defined by $v$ is:

$$
\left.\left.\left.S_{\eta}=S+v_{\eta}=S+\sum_{r=1}^{\infty} \frac{1}{(2 r) !} v^{2 r}\right\lrcorner \eta\right\lrcorner \cdots\right\lrcorner \eta
$$

so that the extended action satisfies:

$$
\hat{v}_{\eta}\left(S_{\eta}\right)=-\frac{1}{2}[v, v]_{\eta}
$$

More terms must be added to $v$, usually constructed from the algebra the symmetries form, so that it satisfies the classical master equation, in which case the extended action $S_{\eta}$ would be symmetric under $\hat{v}_{\eta}$.

\subsection{The functional integral}

The classical action determines the phases for a quantum field theory. The Green's functions are given by:

$$
\left\langle e^{J}\right\rangle=Z[J] / Z[0]
$$

where $J$ is a local functional and $Z[J]$ is the functional integral:

$$
Z[J]=\int[d \phi] e^{\frac{i}{\hbar} S[\phi]+J[\phi]}
$$


The functional integral is a formal integral over the space $\Gamma(\pi)$ of sections, and must be regularised before the measure $[d \phi]$ may be defined. The regularisation is achieved by modifying the classical action $S[\phi]$ with a regulator local functional $S_{\text {reg }}[\phi ; \Lambda]$, vanishing as the regularisation is removed, $\Lambda \rightarrow \infty$. The regularised functional integral:

$$
Z[0 ; \Lambda]=\int[d \phi] e^{\frac{i}{\hbar}\left(S[\phi]+S_{\mathrm{reg}}[\phi ; \Lambda]\right)}
$$

is defined for each value of the regularisation parameter $\Lambda$. A candidate for the partition function $Z[0]$ is given by the $\operatorname{limit}_{\Lambda \rightarrow \infty} Z[0 ; \Lambda]$. However this limit may be divergent, and the limiting procedure must be accompanied by a renormalisation of the action in order to achieve a finite result for $Z[0]$.

In the perturbation theory on linear bundles, the coefficient fields $S_{\alpha}{ }_{\beta_{1} \ldots \beta_{n}}^{I_{1} \ldots I_{n}}$ appearing in the expansion of the action:

$$
S\left[\phi_{0}+\phi\right]=S\left[\phi_{0}\right]+\sum_{n=1}^{\infty} \frac{1}{(n+1) !} \int \phi^{\alpha} \phi_{I_{1}}^{\beta_{1}} \ldots \phi_{I_{n}}^{\beta_{n}} S_{\alpha \beta_{1} \ldots \beta_{n}}^{I_{1} \ldots I_{n}} \Omega
$$

about a background solution $\phi_{0}$ of the classical field equation determine the Feynman rules for the quantum field theory円. The requirement for regularisation manifests itself in the need to regularise graphs with short distance (i.e. UV) divergences. The coefficient fields are renormalised with $O(\hbar)$ counterterms, divergent as the regularisation is removed, so that the $\operatorname{limit}_{\Lambda \rightarrow \infty} Z[0 ; \Lambda]$ is finite.

The renormalisation procedure must maintain the locality of the action, i.e. the counterterms must not contain arbitrarily high order derivatives. This cannot be guaranteed for the general local functional, as the counterterms are dictated by the need to renormalise divergent graphs. Thus only certain renormalisable local functionals may be taken as classical actions, determined by the power counting rules.

To define the functional integral $Z[J]$, and hence define the Green's function for the local functional operator $J$, extra counterterms must be added to renormalise the graphs that include $J$-insertions. In order to make it clear that the operator is to be understood only in the renormalised sense, use the normal product notation $N_{d}(J)$, where $d$ is the UV dimension of $J$. The ambiguity in the renormalisation procedure allows for finite renormalisations of the normal product $N_{d}$ - for a basis of local functional operators $\left\{J_{i}\right\}$ with UV degree

\footnotetext{
${ }^{1}$ For nonlinear bundles, the bundle must be linearised before this analysis may be performed. This may for instance be achieved by using geodesic coordinates, when the space $B$ is endowed with a connection. For a background field $\phi_{0}$ the quantum field is a vertical section $v$ of the tangent bundle to $B$ at $\phi_{0}, v \in \Gamma\left(\phi_{0}^{*} V B \rightarrow M\right)$. The vertical tangent field $v$ defines geodesic deformations $e^{v}$ of the background field $\phi_{0}$, and the action $S[\phi]$ defines a local functional $S\left[e^{v} \phi_{0}\right]$ on the bundle $\phi_{0}^{*} V B \rightarrow M$. This functional may be used to generate the Feynman rules.
} 
$d_{i} \leq d$, an alternative normal product is given by:

$$
\tilde{N}_{d}(J)=N_{d}\left(J+\sum_{i} c_{i} J_{i}\right)
$$

with $O(\hbar)$ coefficients $c_{i}$. The renormalisation procedure frequently causes operator expressions whose classical analogues vanish not to be zero, but to be given by $O(\hbar)$ anomalous operators. The above arbitrariness in the normal product may then be employed in an attempt to remove the anomalies.

Ward identities reflecting the presence of symmetries in the quantum field theory may be generated from the formal Stokes' theorem for the functional integral:

$$
\begin{aligned}
0 & =\int[d \phi] v\left(e^{\frac{i}{\hbar} S[\phi]+J[\phi]}\right) \\
& =\int[d \phi] e^{\frac{i}{\hbar} S[\phi]+J[\phi]}\left(\frac{i}{\hbar} v S+v J\right)[\phi]
\end{aligned}
$$

so that, if the action $S$ is invariant under the evolutionary vector field $v, v S=0$, then the Ward identity for the local functional $J$ is:

$$
0=\langle v J\rangle
$$

The renormalisation of the action and the symmetry cause extra anomalous insertions in the above expression. In fact the validity of the Ward identity relies on two conditions:

1. Invariance of the regulator: The regularised functional integral includes the regulator term $S_{\text {reg. }}$. Thus at the regularised stage the expression above should read:

$$
0=\int[d \phi] e^{\frac{i}{\hbar}\left(S[\phi]+S_{\mathrm{reg}}[\phi ; \Lambda]\right)+J[\phi]}\left(\frac{i}{\hbar} v\left(S+S_{\mathrm{reg}}\right)+v J\right)[\phi ; \Lambda]
$$

resulting in an anomalous insertion after the limit $\Lambda \rightarrow \infty$ is taken, due to the renormalisation of the action when the regulator is not invariant under the symmetry.

2. Invariance of the measure: It cannot be guaranteed that the regularised measure $[d \phi]$ is invariant under the symmetry, implicitly assumed in the application of the formal Stokes' theorem above. In general, the regularised divergence $\Delta v$ is non-zero, and this results in an anomalous insertion after the regularisation is removed, given by the renormalised divergence of the symmetry. The renormalised divergence operator $\Delta$ defines a map from the space of evolutionary vector fields $\Sigma^{1} \pi$ to the space of local functionals $\Sigma_{0} \pi$. 
The renormalised Ward identity is thus:

$$
0=\langle v J\rangle+\frac{i}{\hbar}\langle J \Delta\rangle
$$

where the anomalous insertion $\Delta$ is due to the renormalisation of the action and the divergence of the symmetry.

An even functional multivector $\Gamma$ may be functionally integrated after repeated contraction with fermionic functional 1-forms, so that each fermionic functional 1-form $\eta \in \Sigma_{1} \pi$ defines the Green's functions for a quantum field theory:

$$
\left\langle e^{J}\right\rangle_{\eta}=Z_{\eta}[J] / Z_{\eta}[0]
$$

where $Z_{\eta}[J]$ is the functional integral:

$$
Z_{\eta}[J]=\int[d \phi] e^{\frac{i}{\hbar} \Gamma_{\eta}[\phi]+J[\phi]}
$$

The definition above is dependent on the renormalisability of the action $\Gamma_{\eta}$. In general, the quantum field theories are defined only on a subset of the fermionic functional 1-forms, determined by the even functional multivector action $\Gamma$.

Consider the dependence of the functional integral on the fermionic functional 1 -form. Under a perturbation $\chi$ of $\eta$, the action $\Gamma_{\eta}$ changes by $\left.\hat{\Gamma}_{\eta}\right\lrcorner \chi$, to lowest order in $\chi$. Thus:

$$
\left.Z_{\eta+\chi}[J]-Z_{\eta}[J]=\frac{i}{\hbar} \int[d \phi] e^{\frac{i}{\hbar} \Gamma_{\eta}[\phi]+J[\phi]}\left(\hat{\Gamma}_{\eta}\right\lrcorner \chi\right)[\phi]
$$

to lowest order in $\chi$. If the perturbation is exact, $\chi=\delta \psi$, the perturbation of $\Gamma_{\eta}$ is $\hat{\Gamma}_{\eta}(\psi)$, so that 'integration by parts' may be performed in the functional integral:

$$
Z_{\eta+\delta \psi}[J]-Z_{\eta}[J]=-\frac{i}{\hbar} \int[d \phi] e^{\frac{i}{\hbar} \Gamma_{\eta}[\phi]+J[\phi]}\left(\frac{i}{\hbar} \hat{\Gamma}_{\eta}\left(\Gamma_{\eta}\right)+\hat{\Gamma}_{\eta}(J)\right)[\phi] \psi[\phi]
$$

The important case is when the fermionic functional 1-form is closed, $\eta \in \operatorname{Ker} \delta \cap$ $\Sigma_{1} \pi$, so that:

$$
Z_{\eta+\delta \psi}[J]-Z_{\eta}[J]=\frac{i}{\hbar} \int[d \phi] e^{\frac{i}{\hbar} \Gamma_{\eta}[\phi]+J[\phi]}\left(\frac{i}{2 \hbar}[\Gamma, \Gamma]_{\eta}+[\Gamma, J]_{\eta}\right)[\phi] \psi[\phi]
$$

explicitly displaying the relation between the functional integral and the classical master equation. Thus if $\Gamma$ satisfies the classical master equation, then the Green's functions for symmetric operators depend only on the cohomology class of the closed fermionic functional 1-form $\eta$ in the variational cohomology group $H_{1}(\pi, \delta)$. This result is important for two reasons, explicitly demonstrated in later sections. Firstly, this expression generates the Lam action principle for the 
quantum effective action, and hence generates the anomaly consistency condition. Secondly, when the action exhibits gauge symmetries, and the propagators are undefined, the 'gauge fixed' extension $\Gamma_{\eta}$ may be used instead of the original action. The above expression gives the dependence of the resulting quantum field theory on the fermionic gauge fixing functional 1-form $\eta$.

The validity of the above formal manipulation of the functional integral requires a renormalisation procedure that does not introduce anomalous insertions, i.e. the regulator and measure must be invariant:

1. Invariance of the regulator: The functional integral is regularised with an even functional multivector regulator $\Gamma_{\text {reg }}$ (this allows for actions $\Gamma_{\eta}$ defined by different fermionic functional 1-forms $\eta$ to be regularised with different regulators $\left.\Gamma_{\operatorname{reg} \eta}\right)$. Thus even when the action $\Gamma$ satisfies the classical master equation, the regularised action $\Gamma+\Gamma_{\text {reg }}$ may not, and residual anomalous odd functional multivector insertions may remain after the regularisation is removed.

2. Invariance of the measure: The formal manipulations in the regularised functional integral must include divergence terms when the measure is not invariant under the evolutionary vector field $\hat{\Gamma}_{\eta}$. The renormalised divergence operator $\Delta$ on evolutionary vector fields defines a divergence operator $\Delta$ on the functional multivectors, given by:

$$
(\Delta v)_{\eta}=\Delta\left(\hat{v}_{\eta}\right)
$$

The divergence operator is effectively a coderivative to the variational derivative, mapping down the functional multivectors:

$$
\cdots \quad \Sigma^{s+1} \pi \quad \stackrel{\Delta}{\rightarrow} \quad \Sigma^{s} \pi \quad \cdots \quad \Sigma^{1} \pi \quad \stackrel{\Delta}{\rightarrow} \quad \Sigma^{0} \pi \quad \rightarrow \quad 0
$$

given by the renormalised trace $\Delta v=\operatorname{tr}(\delta v)$. Note however that $\Delta$ is not necessarily nilpotent, in general $\Delta^{2}$ will be proportional to the renormalised Ricci tensor on the space of sections $\Gamma(\pi)$.

Both these factors result in a possible anomalous odd functional multivector insertion $\Delta$ in the formal expressions above, and may destroy the independence of the Green's functions on the fermionic functional 1-form.

The even functional multivector $v=\sum_{r=1}^{\infty} v^{2 r}$ defines extensions to the classical action, and each extended action determines the phases for a quantum field theory. Proceeding as before, consider the functional integral:

$$
Z_{\eta}[J]=\int[d \phi] e^{\frac{i}{\hbar} S_{\eta}[\phi]+J[\phi]}
$$

related to the Green's functions via:

$$
Z_{\eta}[J]=Z_{\eta}[0]\left\langle e^{J}\right\rangle_{\eta}
$$


If $\eta$ is a closed fermionic functional 1 -form, and $\psi$ is a fermionic local functional, then to lowest order in $\psi$ :

$$
Z_{\eta+\delta \psi}[J]-Z_{\eta}[J]=\frac{i}{\hbar} \int[d \phi] e^{\frac{i}{\hbar} S_{\eta}[\phi]+J[\phi]}\left(\frac{i}{2 \hbar}[v, v]_{\eta}+[v, J]_{\eta}\right)[\phi] \psi[\phi]
$$

Thus if the even functional multivector $v$ can be modified to solve the classical master equation at $\eta,[v, v]_{\eta}=0$, then the Green's functions for functionals symmetric under $v$ at $\eta$ are unchanged by exact perturbations of $\eta$. As before, renormalisation introduces extra anomalous terms into the above expression, due to regulators for both the action $S$ and the even functional multivector $v$, and the possible non-vanishing divergence of $v$.

The analysis above can be repeated in the field-antifield formalism when the bundle $B \stackrel{\pi}{\rightarrow} M$ is a vector bundle (more generally, the analysis applies locally for a nonlinear bundle when it is linearised). Take the fermionic functional 1form to be field independent, $\eta=\int \eta_{\alpha}(x) d \phi^{\alpha} \wedge \Omega$ for a fermionic antifield $\eta \in$ $\Gamma\left(B^{*} \rightarrow M\right)$. Functional multivectors are then identified with their contracted counterparts, local functionals of the field $\phi$ and its antifield $\eta$.

Define the generating functional for the Green's functions:

$$
Z[j]=\int[d \phi] e^{\frac{i}{\hbar} S[\phi]+\int j_{\alpha} \phi^{\alpha} \Omega}
$$

Ward identities corresponding to the symmetry of the action $S$ under the evolutionary vector field $v$ are generated from the formal functional integral expression:

$$
0=\int[d \phi] e^{\frac{i}{\hbar} S[\phi]+\int j_{\alpha} \phi^{\alpha} \Omega}\left(\int j_{\alpha} v^{\alpha} \Omega\right)
$$

so that the Ward identity for the $n$-point Green's function is:

$$
0=\frac{i}{\hbar}\left\langle\phi^{\alpha_{1}}\left(x_{1}\right) \ldots \phi^{\alpha_{n}}\left(x_{n}\right) \Delta\right\rangle+\sum_{r=1}^{n}\left\langle\phi^{\alpha_{1}}\left(x_{1}\right) \ldots v^{\alpha_{r}}\left(x_{r}\right) \ldots \phi^{\alpha_{n}}\left(x_{n}\right)\right\rangle
$$

where the presence of the anomalous insertion $\Delta$ reflects the possible lack of invariance of the measure and the regulator under the symmetry.

For the even functional multivector action $\Gamma$, construct the generating functional in the presence of the external antifield:

$$
Z[j, \eta]=\int[d \phi] e^{\frac{i}{\hbar} \Gamma[\phi, \eta]+\int j_{\alpha} \phi^{\alpha} \Omega}
$$

The dependence on the antifield may be analysed:

$$
\begin{aligned}
\int j_{\alpha} \frac{\delta Z}{\delta \eta_{\alpha}} \Omega & =\frac{i}{\hbar} \int[d \phi] e^{\frac{i}{\hbar} \Gamma[\phi, \eta]}\left\{\int \Omega \frac{\delta \Gamma}{\delta \eta_{\alpha}} \frac{\partial}{\partial \phi^{\alpha}}\right\}\left(e^{\int j_{\alpha} \phi^{\alpha} \Omega}\right) \\
& =\frac{1}{\hbar^{2}} \int[d \phi] e^{\frac{i}{\hbar} \Gamma[\phi, \eta]+\int j_{\alpha} \phi^{\alpha} \Omega}\left(\int \frac{\delta \Gamma}{\delta \eta_{\alpha}} \frac{\delta \Gamma}{\delta \phi^{\alpha}} \Omega\right)
\end{aligned}
$$


If the even functional multivector $\Gamma$ satisfies the classical master equation, then the generating functional satisfies the Lam action principle:

$$
\int j_{\alpha} \frac{\delta Z}{\delta \eta_{\alpha}} \Omega=0
$$

The introduction of an even functional multivector regulator $\Gamma_{\text {reg }}$ and the lack of invariance of the measure introduce extra anomalous insertions into the above expression. The renormalised Lam action principle for the generating functional is thus:

$$
\int j_{\alpha} \frac{\delta Z}{\delta \eta_{\alpha}} \Omega=\frac{1}{\hbar^{2}} \Delta \circ Z
$$

where $\Delta$ is an odd functional multivector insertion.

The quantum effective action $\Gamma_{q}$ is constructed by taking the Legendre transform of the (connected) generating functional with respect to the source field $j$, and is a non-local even functional multivector. By taking the Legendre transform of the Lam action principle for the generating functional, the Lam action principle for the quantum effective action is seen to be:

$$
\left[\Gamma_{q}, \Gamma_{q}\right]=\Delta \circ \Gamma_{q}
$$

For a general bundle $B \stackrel{\pi}{\rightarrow} M$, the quantum effective action $\Gamma_{q}$ at the section $\phi$ may be constructed from a linear approximation to the bundle about $\phi$. With this definition of the quantum effective action, the Lam action principle above remains valid for nonlinear bundles.

\subsection{Anomalies and the consistency condition}

Consider the consequences of the Lam action principle:

$$
\left[\Gamma_{q}, \Gamma_{q}\right]=\Delta \circ \Gamma_{q}
$$

for the anomalous odd functional multivector insertion $\Delta$. Take the commutator with $\Gamma_{q}$ - the Jacobi identity for the Schouten bracket gives:

$$
\left[\Gamma_{q}, \Delta \circ \Gamma_{q}\right]=0
$$

It is observed in the perturbation theory that the quantum effective action may be constructed by summing over the 1-particle irreducible graphs. Thus in particular, in the $\hbar$-expansion of $\Gamma_{q}$ :

$$
\Gamma_{q}=\sum_{r=0}^{\infty} \hbar^{r} \Gamma_{q r}
$$

the first term is given by the classical action, $\Gamma_{q 0}=\Gamma$. Furthermore, if the anomalous insertion is expanded as $\Delta=\hbar^{r} \Delta_{r}+O\left(\hbar^{r+1}\right)$, then:

$$
\Delta \circ \Gamma_{q}=\hbar^{r} \Delta_{r}+O\left(\hbar^{r+1}\right)
$$


The lowest order term in the $\hbar$-expansion of the expression $\left[\Gamma_{q}, \Delta \circ \Gamma_{q}\right]=0$ is:

$$
\left[\Gamma, \Delta_{r}\right]=0
$$

This is the consistency condition for the anomaly - the lowest order anomaly term must be symmetric under $\Gamma$.

The case of interest is when the classical action $S$ is extended by an even functional multivector $v=\sum_{r=1}^{\infty} v^{2 r}$ satisfying $[v, S]=0$, and in particular when $v$ may be taken to be a solution of the classical master equation $[v, v]=0$. The lowest order anomaly must thus be $\Gamma$-closed, $\left[\Gamma, \Delta_{r}\right]=0$, where $\Gamma=S+$ $v$. Suppose the anomaly is $\Gamma$-exact, $\Delta_{r}=\left[\Gamma, \Gamma_{r}\right]$ for some even functional multivector $\Gamma_{r}$. A finite renormalisation of the extended action $\Gamma$ can be used to remove the anomaly:

$$
\Gamma \rightarrow \Gamma-\frac{1}{2} \hbar^{r} \Gamma_{r}
$$

so that $\left[\Gamma_{q}, \Gamma_{q}\right]=O\left(\hbar^{r+1}\right)$. Thus the anomaly is non-trivial if and only if it defines a non-trivial cohomology class in the odd $\Gamma$-cohomology group $H^{\text {odd }}(\pi, \Gamma)$.

\subsection{Gauge fixing}

As an example of the use of the jet formalism, consider the case of a classical action with gauge symmetries. The gauge symmetries define degeneracies in the equations of motion, and prevent the propagators from being defined. The action must be gauge fixed to remove the degeneracies. An obvious possibility is to use an even functional multivector extension, and consider the dependence of the resulting quantum field theories on the gauge fixing fermionic functional 1 -form.

The gauge symmetries are parametrised by fields from a vector bundle, necessarily the collection of fields must be extended to include the parameter field. However, the analysis proceeds as before without requiring quantisation of the parameter field. In fact, in order to generate the Faddeev-Popov determinant in the functional integral, it is the antifield to the parameter field, the ghost field, that must be quantised. This does not invalidate the analysis of previous sections for this case, as the symmetry between the field and its antifield in the Batalin-Vilkovisky antibracket may be exploited. Effectively, the ghost field is treated as the field, with the parameter field appearing as the antifield to the ghost field.

In addition to the ghost field, there is the antifield to the $\phi$-field. However, in the general nonlinear bundle case the antifield cannot be defined. The solution to this is to work with the gauge fixing fermion in place throughout the calculation. The gauge fixing fermion thus appears naturally as the $\phi$-component of the fermionic functional 1-form.

Consider the classical action $S$ with gauge symmetries:

$$
v^{R \alpha} \frac{\delta S}{\delta \phi^{\alpha}}=0
$$


for some coefficient operators $v^{R \alpha}=v^{R \alpha I} d_{I}$. The transitions for the $R$-index of $v^{R \alpha}$ determine a bundle $E \stackrel{\tau}{\rightarrow} M$, with adapted local coordinates $\left(x^{i}, b^{R}\right)$ on $E$ ?. The gauge symmetry expression may be written in functional multivector notation as $[v, S]=0$, where $v$ is the functional bivector:

$$
v=\int \Omega \frac{\partial}{\partial b^{S}} \wedge v^{S \alpha} \frac{\partial}{\partial \phi^{\alpha}}
$$

in $\Sigma^{2}(\pi \times \tau)$. Thus $\left.v\right\lrcorner \omega$ is a symmetry of the action for any functional 1-form $\omega \in \Sigma_{1}(\pi \times \tau)$.

Assume the bundle $E \stackrel{\tau}{\rightarrow} M$ is a vector bundle. Restrict attention to fermionic functional 1-forms $\eta$ of the form:

$$
\eta=\int c_{S} d b^{S} \wedge \Omega+\delta \Psi
$$

for the fermionic ghost field $c \in \Gamma\left(E^{*} \rightarrow M\right)$ and the gauge fixing fermion $\Psi \in \Sigma_{0} \pi$. The action $S$ may be extended by the contracted functional bivector $v_{\eta}$, giving an action dependent on the ghost field as well as the $\phi$-field (but independent of the $b$-field):

$$
S_{\Psi}[\phi, c]=S[\phi]+\int c_{S} v^{S \alpha} \frac{\delta \Psi}{\delta \phi^{\alpha}} \Omega
$$

Consider the functional integral:

$$
Z_{\Psi}[J ; c]=\int[d \phi] e^{\frac{i}{\hbar} S_{\Psi}[\phi, c]+J[\phi]}
$$

for the local functional $J \in \Sigma_{0} \pi$. Analyse the dependence of $Z_{\Psi}[J ; c]$ on the gauge fixing fermion $\Psi$. For the fermionic local functional $\tilde{\Psi} \in \Sigma_{0} \pi$ :

$$
\begin{aligned}
Z_{\Psi+\tilde{\Psi}}[J ; c]-Z_{\Psi}[J ; c]= & \frac{i}{\hbar} \int[d \phi] e^{\frac{i}{\hbar} S_{\Psi}[\phi, c]+J[\phi]} \\
& \times\left(\frac{i}{2 \hbar}[v, v]_{\eta}+[v, J]_{\eta}\right)[\phi, c] \tilde{\Psi}[\phi]
\end{aligned}
$$

to lowest order in $\tilde{\Psi}$ (ignoring anomalous terms). Care must be taken when proving this statement. Since functional integration over the $b$-field is not included in the functional integral, the above expression does not follow directly from the expressions of previous sections. In fact the lack of functional integration over the $b$-field does not introduce extra terms since the ghost field, the gauge fixing fermion, and the extended action are all independent of the $b$-field.

\footnotetext{
${ }^{2}$ For example, in the case of the Yang-Mills theory based on a principal fibre bundle $P \rightarrow M$ with Lie group $G$, the bundle $E \stackrel{\tau}{\rightarrow} M$ is the associated bundle with fibre the Lie algebra $g$ of $G$ in the adjoint representation, and the gauge symmetry operator $v^{R \alpha}$ is given by the covariant derivative.
} 
The extended action determines a gauge fixed quantum field theory. Take the gauge fixing fermion $\Psi$ in the form:

$$
\Psi=\int f_{S}(\phi) \bar{c}^{S} \Omega
$$

for the fermionic antighost field $\bar{c} \in \Gamma(E \stackrel{\tau}{\rightarrow} M)$ and the gauge fixing functions $f_{S}$. If the ghost and antighost fields are quantised, i.e. the functional integral is taken to be:

$$
Z_{f}[J]=\int[d \phi][d c][d \bar{c}] e^{\frac{i}{\hbar} S_{\Psi}[\phi, c, \bar{c}]+J[\phi]}
$$

then the functional integral formally contains the Faddeev-Popov determinant:

$$
\begin{aligned}
Z_{f}[J] & =\int[d \phi] e^{\frac{i}{\hbar} S[\phi]+J[\phi]}\left(\int[d c][d \bar{c}] e^{\frac{i}{\hbar} \int c_{R} v^{R \alpha} E_{\alpha}\left(f_{T} \bar{c}^{T}\right) \Omega}\right) \\
& =\int[d \phi] \delta\left(f_{S}(\phi)\right) e^{\frac{i}{h} S[\phi]+J[\phi]}
\end{aligned}
$$

corresponding to the gauge-fixing conditions $f_{S}=0$.

For $h, g \in \Sigma_{0}(\pi \times \tau)$, the commutator $\left.\left.[v\lrcorner \delta h, v\right\lrcorner \delta g\right]$ is also a symmetry of the action. Suppose the algebra of symmetries generated by $\Sigma_{0}(\pi \times \tau)$ closes on $\Sigma_{0}(\pi \times \tau)$. This defines a Poisson bracket on $\Sigma_{0}(\pi \times \tau)$ :

$$
[v\lrcorner \delta h, v\lrcorner \delta g]=v\lrcorner \delta\{h, g\}
$$

given by a nilpotent functional bivector $\Gamma$ via $\{h, g\}=\Gamma\lrcorner \delta h\lrcorner \delta g$. By counting the powers of the fields in the defining expression above, the algebra functional bivector $\Gamma$ must take the form:

$$
\Gamma=\frac{1}{2} \int \Omega \frac{\partial}{\partial b^{R}} \wedge b_{I}^{S} D_{S}^{R T_{S}^{I}} \frac{\partial}{\partial b^{T}}
$$

for some $b$-independent operator $D^{R T_{S}^{I}}$ (necessarily dependent on the $\phi$-field when the bundle $B \stackrel{\pi}{\rightarrow} M$ is nonlinear $\left.{ }^{3}\right)$.

Consider the consequences of the closure of the algebra for the contracted functional multivectors. The algebra may be written in terms of the evolutionary vector fields $\hat{v}_{\eta}$ as:

$$
\left[\hat{v}_{\eta}, \hat{v}_{\eta}\right]=2\left[v, \Gamma_{\eta}\right]
$$

\footnotetext{
${ }^{3}$ This is a significant generalisation of the Yang-Mills case, where the algebra is given by the functional bivector:

$$
\Gamma=\frac{1}{2} \int \Omega \frac{\partial}{\partial b^{R}} \wedge b^{S} f_{S}^{R T} \frac{\partial}{\partial b^{T}}
$$

where the coefficients $f_{S}^{R T}$ are the structure constants of the Lie algebra $g$. Thus in the YangMills case the operator $D^{R T} T_{S}^{I}$ is a constant, field independent matrix operator, vanishing for $I \neq 0$.
} 
This expression is contracted with $\eta$ to get a local functional expression:

$$
[v, v]_{\eta}=\hat{v}_{\eta}\left(\Gamma_{\eta}\right)
$$

Combine this with the expression $[\Gamma, \Gamma]_{\eta}=0$ for the nilpotency of $\Gamma$ to obtain:

$$
\left[v+\frac{1}{2} \Gamma, v+\frac{1}{2} \Gamma\right]_{\eta}+\hat{\Gamma}_{\eta}\left(v_{\eta}\right)=0
$$

The functional bivector $v+\frac{1}{2} \Gamma$ is thus nilpotent on fermionic functional 1-forms for which $\hat{\Gamma}_{\eta}\left(v_{\eta}\right)$ vanishes. In particular, for fermionic functional 1-forms of the form $\eta=\int c_{S} d b^{S} \wedge \Omega+\delta \Psi$ the local functional $v_{\eta}$ is independent of the $b$-field. Thus $\hat{\Gamma}_{\eta}\left(v_{\eta}\right)=0$, and hence $v+\frac{1}{2} \Gamma$ satisfies the classical master equation at $\eta$.

In the case of a closed algebra, include a term dependent on the external $b$-field in the extended action. For $\eta=\int c_{S} d b^{S} \wedge \Omega+\delta \Psi$, the extended action becomes:

$$
\begin{aligned}
S_{\Psi}[\phi, c ; b] & =S[\phi]+v_{\eta}[\phi, c]+\frac{1}{2} \Gamma_{\eta}[\phi, c ; b] \\
& =S[\phi]+\int c_{S} v^{S \alpha} \frac{\delta \Psi}{\delta \phi^{\alpha}} \Omega+\frac{1}{4} \int c_{R} b_{I}^{S} D^{R T^{I}}{ }_{S} c_{T} \Omega
\end{aligned}
$$

Consider the functional integral:

$$
Z_{\Psi}[J ; b]=\int[d \phi][d c] e^{\frac{i}{\hbar} S_{\Psi}[\phi, c ; b]+J[\phi]}
$$

obtained by quantising the ghost field, leaving the $b$-field as an external field (this reversal of the roles of the $b$ - and $c$-fields is necessary since the extended action is linear in the $b$-field, and hence there are difficulties in defining the functional integration over the $b$-field). The symmetry between the $b$ - and $c$-fields in the Batalin-Vilkovisky antibracket is now exploited to generate the expression for the dependence of the functional integral on the gauge fixing fermion. For $\eta=\int c_{S} d b^{S} \wedge \Omega+\delta \Psi$, the expression above for the bracket $\left[v+\frac{1}{2} \Gamma, v+\frac{1}{2} \Gamma\right]_{\eta}$ is written out in full:

$$
\begin{aligned}
0 & =\left(-2 \int \Omega c_{S} v^{S \alpha} \frac{\partial}{\partial \phi^{\alpha}}\right)\left(v_{\eta}+\frac{1}{2} \Gamma_{\eta}\right)+2 \int \frac{\delta\left(v_{\eta}+\frac{1}{2} \Gamma_{\eta}\right)}{\delta c_{S}} \frac{\delta\left(v_{\eta}+\frac{1}{2} \Gamma_{\eta}\right)}{\delta b^{S}} \Omega \\
& =\int \Omega\left\{-2 c_{S} v^{S \alpha} \frac{\partial}{\partial \phi^{\alpha}}+\frac{1}{2}\left(c_{R} D^{R T_{S}^{I}} c_{T}\right) d_{I} \frac{\partial}{\partial c_{S}}\right\}\left(v_{\eta}+\frac{1}{2} \Gamma_{\eta}\right)
\end{aligned}
$$

The above expression fan now be placed under the functional integral sign,

\footnotetext{
${ }^{4}$ This expression contains the evolutionary vector field:

$$
\int \Omega\left\{c_{S} v^{S \alpha} \frac{\partial}{\partial \phi^{\alpha}}-\frac{1}{4}\left(c_{R} D^{R T}{ }_{S}^{I} c_{T}\right) d_{I} \frac{\partial}{\partial c_{S}}\right\}
$$

corresponding to the BRST variations of the $\phi$ - and $c$-fields in the Yang-Mills theory.
} 
and manipulating the functional integral as before gives:

$$
Z_{\Psi+\tilde{\Psi}}[J ; b]-Z_{\Psi}[J ; b]=\frac{i}{\hbar} \int[d \phi][d c] e^{\frac{i}{\hbar} S_{\Psi}[\phi, c ; b]+J[\phi]}[v, J]_{\eta}[\phi, c] \tilde{\Psi}[\phi]
$$

to lowest order in $\tilde{\Psi}$ (neglecting anomaly terms). It should be clear from this example that the formal expressions of the previous sections have analogous expressions when the roles of the fields and the antifields are reversed. The functional integral, where defined, is thus independent of the gauge fixing fermion for symmetric sources $J$ (in the absence of anomaly terms). In particular, if the physical operators are required to be symmetric, then the quantum field theories constructed from different gauge fixing fermions are equivalent. 


\section{Acknowledgements}

Thanks to Paul Howe for his encouragement with this article. This research is funded by the Science and Engineering Research Council.

\section{References}

[1] Peter J. Olver. Applications of Lie Groups to Differential Equations, volume 107 of Graduate Texts in Mathematics. Springer-Verlag, 1986.

[2] D. J. Saunders. The Geometry of Jet Bundles, volume 142 of London Mathematical Society Lecture Note Series. Cambridge University Press, 1989.

[3] I. A. Batalin and G. A. Vilkovisky. Gauge algebra and quantization. Phys. Lett., B102:27-31, 1981.

[4] I. A. Batalin and G. A. Vilkovisky. Quantization of gauge theories with linearly dependent generators. Phys. Rev., D28:2567-2582, 1983.

[5] Peter J. Olver. On the Hamiltonian structure of evolution equations. Math. Proc. Camb. Phil. Soc., 88:71-88, 1980.

[6] F. Magri. A simple model of the integrable Hamiltonian equation. J. Math. Phys., 19:1156-1162, 1978.

[7] I. M. Gel'fand and I. Ya. Dorfman. Hamiltonian operators and algebraic structures related to them. Func. Anal. Appl., 13:248-262, 1979.

[8] I. M. Gel'fand and I. Ya. Dorfman. The Schouten bracket and Hamiltonian operators. Func. Anal. Appl., 14:71-74, 1980.

[9] I. M. Gel'fand and I. Ya. Dorfman. Hamiltonian operators and infinitedimensional Lie algebras. Func. Anal. Appl., 15:173-187, 1981.

[10] Andre Lichnerowicz. Les variétés de Poisson et leurs algebres de Lie associées. J. Diff. Geom., 12:253-300, 1977.

[11] Andre Lichnerowicz. Variétés de poisson et feuilletages. Ann. Fac. Sci. Toulouse, 4:195-262, 1982.

[12] I. A. Batalin and I. V. Tyutin. Quantum geometry of symbols and operators. Nucl. Phys., B345:645-658, 1990.

[13] I. A. Batalin and I. V. Tyutin. On possible generalizations of field-antifield formalism. Preprint FIAN-TD-18-92, 1992. 
[14] Rafael Ferraro, Marc Henneaux, and Marcel Puchin. On the quantization of reducible gauge systems. Preprint ULB-PMIF-92-07, 1992.

[15] Phillipe Grégoire and Marc Henneaux. Hamiltonian BRST-anti-BRST theory. Preprint ULB-PMIF-92-06, 1992.

[16] Marc Henneaux. Geometric interpretation of the quantum master equation in the BRST-anti-BRST formalism. Phys. Lett., B282:372-376, 1992.

[17] Y. M. P. Lam. Phys. Rev., D6:2145,2161, 1972.

[18] T. E. Clark and J. H. Lowenstein. Nucl. Phys., B133:109, 1976.

[19] O. Piguet and A. Rouet. Symmetries in perturbative quantum field theory. Phys. Reports, 76:1-77, 1981.

[20] A. Diaz, W. Troost, P. van Nieuwenhuizen, and A. Van Proeyen. Understanding Fujikawa regulators from Pauli-Villars regularization of ghost loops. Int. J. Mod. Phys., 4A:3939-3982, 1989.

[21] W. Troost, P. van Nieuwenhuizen, and A. Van Proeyen. Anomalies and the Batalin-Vilkovisky lagrangian formalism. Nucl. Phys., B333:727-770, 1990.

[22] P. S. Howe, U. Lindström, and P. White. Anomalies and renormalisation in the BRST-BV framework. Phys. Lett., B246:430-434, 1990. 